

\title{
EB Erwachsenenbildung
}

\author{
KEB \\ Vierteljahresschrift für Theorie und Praxis \\ Heft 2 | 66. Jahrgang | 2020 \\ ISSN (Printausgabe): 0341-7905, ISSN (online): 2365-4953 \\ DOI 10.3278 / EBZ1503W \\ атно ISCHE (KEB Deutschland) \\ Herausgegeben von der Katholischen Erwachsenenbildung Deutschland - Bundesarbeitsgemeinschaft e.V.

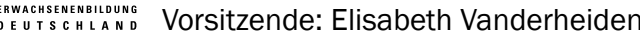

Redaktion: Dr. Marie-Christine Kajewski, Hannover; Dr. Cornelius Sturm, Bonn; Prof. Dr. Ulrich Papenkort, Mainz; Mag.a Martina Bauer (Forum Katholischer Erwachsenenbildung in Österreich), Wien; Dr. Michael Sommer, Mülheim a.d. Ruhr (verantw. Redakteur)

Beirat: Prof. Dr. Ralph Bergold, Siegburg (Vors.); Prof. Dr. Johanna Bödege-Wolf, Vechta; Prof. Dr. Regina Egetenmeyer-Neher, Würzburg; Prof. Dr. Norbert Frieters-Reermann, Aachen; Andrea Heim, Bonn; Prof. Dr. Tetyana Kloubert, Eichstätt; Prof. Dr. Josef Schrader, Bonn; Dr. Hermann Kues, Lingen

Anschrift: Rheinweg 34, 53113 Bonn. Tel.: (02 28) 902 47-0, Fax: (02 28) 902 47-29, Internet: www.keb-deutschland.de, E-Mail: keb@keb-deutschland.de,sommer@redaktion-erwachsenenbildung.de

Bezugsbedingungen: Die Zeitschrift erscheint viermal jährlich. Die Bezugsdauer verlängert sich, wenn das Abonnement nicht bis zum 01.10. gekündigt wird. Die Kündigung ist schriftlich zu richten an: HGV Hanseatische Gesellschaft für Verlagsservice mbH, Leserservice, Holzwiesenstr. 2, 72127 Kusterdingen, E-Mail: v-r-journals@hgv-online.de. Preise und weitere Informationen unter www.vandenhoeck-ruprecht-verlage.com

Beiträge und Zuschriften sind an die Redaktion zu richten. Für unverlangt eingesandte Manuskripte einschließlich Rezensionen wird keine Verantwortung übernommen. Sie gelten erst nach ausdrücklicher Bestätigung als angenommen. Namentlich gezeichnete Beiträge sind Meinungsäußerungen der Autoren und Autorinnen und keine Stellungnahmen des Herausgebers oder der Redaktion. Ohne Aufforderung zugestellte Besprechungsstücke werden nicht zurückgesandt. Ihre Rezension liegt im Ermessen der Redaktion.

(c) 2020, Vandenhoeck \& Ruprecht GmbH \& Co. KG, Theaterstr. 13, 37073 Göttingen. Verantwortlich für die Anzeigen: Anja Kütemeyer, Verlag Vandenhoeck \& Ruprecht. www.vandenhoeck-ruprecht-verlage.com

Alle Rechte vorbehalten. Das Werk und seine Teile sind urheberrechtlich geschützt. Jede Verwertung in anderen als den gesetzlich zugelassenen Fällen bedarf der vorherigen schriftlichen Einwilligung des Verlages.

Druck- und Bindearbeiten: Hubert \& Co. GmbH \& Co. KG BuchPartner, Robert-Bosch-Breite 6, 37079 Göttingen.

Gedruckt auf alterungsbeständigem Papier.

Titelbild: The flower bouquet women. Diese Frau aus Westbengalen lernt traditionelle Blumenbouquets aus Jhuphsi, einer Wildblume aus der Region, zu binden. Foto: CC BY-NC-SA 3.0 IGO (C) UNESCO-UNEVOC/Amitava Chandra 


\section{Thema}

51 Zum Thema: Erwachsenenbildung in und aus aller Welt

52 Katarina Popović

Erwachsenenbildung weltweit auf dem

Rückzug? Trotz ihrer globalen Bedeutung wird die Bildung für Erwachsene vernachlässigt

$56 \quad$ Uwe Gartenschlaeger

Vor neuen Aufgaben. Die europäische

Erwachsenenbildung in unruhigen Zeiten

59 Akihiro Ogawa

Shōgai gakushū - lebenslanges Lernen

in Japan. Gemeinsames Lernen für die

Gemeinschaft und das kulturelle Wachstum

63 Clara Kuhlen, Regina Egetenmeyer

Mehr als Alphabetisierung! Interview mit

Professor/-innen aus Nigeria über die Bedeu-

tung von Erwachsenenbildung

\section{Bildung heute}

68 Erwachsenenbildung ab 2020: Finanzierung verbessern, digitale Kompetenzen fördern. EUBericht: Resümee und Empfehlungen

69 Streit um das künftige Budget von Erasmus+. Geplante Verdoppelung in Gefahr

70 "Experten in eigener Sache“. Projekt zur inklusiven Bildung

71 Älteren fehlen digitale Kompetenzen. Studie der Bertelsmann Stiftung

$72 \quad \mathbf{5 0}$ Jahre Fairer Handel. MISEREOR ruft nach gerechter und ökologischer Wirtschaft

73 Von Entwicklungshilfe zu Agiamondo. Der katholische Entwicklungsdienst AGEH mit neuem Namen / 60. Geburtstag

\section{Aus der KEB}

74 Mehr vom Glauben wissen. Ein Angebot theologischer Erwachsenenbildung feiert sein 50-jähriges Bestehen

75 Rettungsschirm für die Erwachsenenbildung! Stellungnahme der KEB Deutschland

76 Elisabeth Vanderheiden: Freie Lizenzen bei öffentlich-rechtlichen Bildungsinhalten - Ein wichtiger Meilenstein zu mehr freien Bildungsressourcen. Position

\section{Osterreich}

77 Michaela Stauder: "Gemma Demokratie". Menschenrechte als Leitlinie in der Demokratiebildung

\section{Umschau}

79 Bernhard Eder: Weichen stellen, inspiriert - aktiv - engagiert. Erfahrungen eines Bildungsprojekts mit älteren Menschen für eine subjektorientierte Erwachsenenbildung

\section{Praxis}

82 Jennifer Danquah, Stefanie Kröner, Regina Egetenmeyer: Internationale Erwachsenenbildung in verwobenen Welten. Die Winter School der Universität Würzburg

84 Bildung, Dialog, Entwicklung. DVV International ist weltweit aktiv

85 Kirgisistan: DREAM gegen Radikalisierung. Demokratie und Religion - Dialog zwischen gleichberechtigten und gemäßigten Stimmen

86 Kambodscha: Die freiwilligen Lehrkräfte von Ratanakiri. Grundbildung für indigene Gemeinschaften

87 Ökolandwirtschaft: Alle lernen mit. Bildung für nachhaltige Entwicklung in Lateinamerika

88 Bosnien und Herzegowina: Ein System für unüberschaubare Vielfalt. Harmonisierung für ein Land mit 14 Regierungen und 11 Erwachsenenbildungsgesetzen

89 Mali: Zugang zur digitalen Welt. Der Post-LiteracyAnsatz ermöglicht innovative Chancen für Entwicklung und Lernen

\section{Material}

90 Publikationen, Praxishilfen und Internetseiten

93 Rezensionen

\section{Bildserie}

Die Serie Was treiben wir Deutschen in Afrika? zeigt Orginalfotos aus der Zeit um 1914, die die deutsche Kolonialaktivitäten in Afrika dokumentieren. Mehr dazu auf Seite 67 


\title{
Shōgai gakushū - lebenslanges Lernen in Japan
}

\author{
Gemeinsames Lernen für die Gemeinschaft und das kulturelle Wachstum
}

\begin{abstract}
Lebenslanges Lernen oder "shōgai gakushū" nimmt eine wichtige Stellung in der japanischen Bildungslandschaft ein. Dabei hat es Formen entwickelt, die typisch für das Inselreich sind. Dazu gehören nicht nur Inhalte der persönlichen Entwicklung, sondern vor allem Angebote, den gemeinschaftlichen Zusammenhalt im Sozialraum und der Gesellschaft zu fördern. Zentrale Orte der Erwachsenenbildung sind die "kōminkan" - öffentliche Begegnungsräume, die es in jeder Gemeinde gibt.
\end{abstract}

Lebenslanges Lernen bezieht sich in Japan auf eine aktive Form der Bildung. Es umfasst verschiedene Arten von Lernaktivitäten, die sich um persönliche Lernhobbys, Sport und freie Künste drehen. Die Berufsausbildung und die wiederkehrende Fortbildung, die darauf abzielen, die Kenntnisse und Fähigkeiten des Einzelnen für das Überleben auf dem Arbeitsmarkt zu aktualisieren, sind außerdem ein wichtiger Bestandteil des persönlichen Lernens. Die Statistik zeigt, dass fast ein Drittel der japanischen Bevölkerung an einer Art lebenslangem Lernen teilnimmt. Das ist das Ergebnis der festen Zusammenarbeit zwischen Staat und Gesellschaft seit dem Beginn des Zweiten Weltkriegs. Eine der charakteristischen Praktiken, die im japanischen lebenslangen Lernen be-

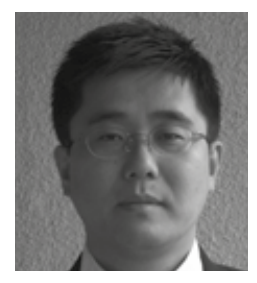

Prof. Dr. Akihiro Ogawa ist ein international tätiger Wissenschaftler. Er hat seit 2015 den Lehrstuhl für Japanstudien an der Universität von Melbourne inne. Er leitet das Forschungsnetzwerk der Asiatischen Zivilgesellschaft und ist Herausgeber des Routledge-Handbuchs der Zivilgesellschaft in Asien (2017). obachtet werden, heißt auf Japanisch "shakai kyōiku" oder wörtlich übersetzt "soziale Erziehung". Shakai kyōiku ist eine gemeinschaftsorientierte Lernpraxis, die alle Arten von Bildung außer der formalen Schulbildung umfasst und sich an alle Generationen von den sehr jungen bis an ältere Menschen richtet. Sie ist informell, beinhaltet aber organisierte Programme, die in lokalen Einrichtungen und nicht in Schulen durchgeführt werden.

\section{Ein Recht auf Lernen}

Diese Formen des Lernens wurden durch das 1949 verabschiedete Sozialerziehungsgesetz (shakai kyōiku hō) unterstützt. Nach diesem Gesetz ist das lebenslange Lernen ein gesetzliches Recht für alle Japaner/-innen. Das Gesetz erkennt an, dass dem Einzelnen eine Fülle von Lernmöglichkeiten zur Entwicklung von Wissen, Fähigkeiten, Gefühlen und körperlichen Fähigkeiten für sein persönliches Wachstum und seine Reife während der gesamten Lebensspanne geboten werden müssen. Das Gesetz war verantwortlich für die bedeutenden Wiederaufbau- und Revitalisierungsbemühungen, um nach dem Krieg ein demokratisches Japan aufzubauen, indem es das Lernen der Bürger/-innen förderte. Darüber hinaus erließ die japanische Regierung 1990 ein Gesetz zur Förderung des lebenslangen Lernens, um das administrative Umfeld für lebenslanges Lernen im beginnenden 21. Jahrhundert vorzubereiten. Das Gesetz schreibt verschiedene Maßnahmen vor, einschließlich der Einrichtung von Räten für lebenslanges Lernen auf nationaler und Präfekturebene.

\section{Kollektives Lernen in lokalen Gemeinschaften}

Diese zwei Gesetze führten zu tief verwurzeltem kollektivem Lernen in den lokalen Gemeinschaften Japans im Rahmen des lebenslangen Lernens. Die Aktivitäten wurden hauptsächlich in öffentlichen Begegnungsräumen für Bürger/-innen durchgeführt, die als "kōminkan" bekannt sind. Die kōminkan ist eine staatliche öffentliche Einrichtung in der Gemeinde. Nach aktueller Statistik (Oktober 2018) gibt es landesweit 13.993 dieser Bürgersäle. Wenn man bedenkt, dass die Zahl der Gemeinden in Japan 1.741 beträgt, hat jede Gemeinde demnach acht solcher Räumlichkeiten.

Auch gemeinnützige Organisationen (NPOs) spielen eine wichtige Rolle bei der Förderung des lebenslangen Lernens an der Basis. Nach dem NPO-Gesetz von 1998 ist die soziale Erziehung der zweitbeliebteste Tätigkeitsbereich. In den letzten Statistiken vom Dezember 2019 haben sich 24.267 NPOs (gegründet nach dem NPO-Gesetz von 1998) in diesem Bereich registriert. Ein Großteil der NPOs im Bereich der sozialen Erziehung wird von den lokalen Regierungen finanziert.

Zu den Lernangeboten in den öffentlichen Sälen und NPOs der Bürger/-in- 
nen gehören Vorträge, Kunstausstellungen, Filmvorführungen und sportliche Treffen. Die Programme in Jugendstudienklassen, Frauenklassen und Elternbildungskursen befassen sich mit soziaIen Fragen, die die heutige Gesellschaft betreffen. Sie bieten auch Programme der freien und bildenden Kunst, einschließlich Literatur, Kunst, Musik, Tanz und Sport. So wurden im Jahr 2017 384.338 Kurse in den Bürgersälen angeboten. Diese Art des Lernens kann als "Kulturmodell" bezeichnet werden, das lebenslanges Lernen für das individuelle kulturelle Wachstum unabdingbar macht. Lebenslanges Lernen ist ein fester Bestandteil des Lebens jedes Einzelnen in Japan. Es zielt darauf ab, das Lernen zum Wohle des Menschen zu fördern und ist darauf ausgerichtet, in der Freizeit kulturelles Wachstum zu erreichen und einen Geschmack für Musik und Sport zu entwickeln, vor allem im Kontext einer alternden Gesellschaft. Die japanische Gesellschaft erlebt derzeit einen Wandel von einer akademisch orientierten Gesellschaft hin zu einer wissensbasierten Wirtschaft.

\section{Hauptaugenmerk der Bildungsreform}

2015 veröffentlichte ich "Lifelong Learning in Neoliberal Japan: Risk, Community, and Knowledge " ${ }^{1}$ (State University of New York Press). Das Buch stellt Japans Politik und Praktiken des lebenslangen Lernens in den 2000er Jahren und darüber hinaus vor. Es dokumentiert die Entwicklung neuen disziplinarischen Wissens, das durch Praktiken des lebenslangen Lernens erzeugt wird, indem es die Neuorganisation der Öffentlichkeit inmitten des Aufstiegs der neoliberalen Politik in Japan zeigt. Tatsächlich ist das lebenslange Lernen das Hauptaugenmerk der japanischen Bildungsreform geworden, und seine zunehmende Bedeutung wurde im Dezember 2006 deutlich, als der japanische Begriff "shōgai gakushū" oder lebenslanges Lernen in die japanische Bildungscharta, das Grundgesetz der Bildung, aufgenommen wurde. Dies war die erste Überarbeitung seit dem Inkrafttreten der Charta im Jahr 1947.

\section{New Public Commons}

Für eine detailliertere Erklärung geht die aktuelle Diskussion über lebenslanges Lernen über den Rahmen des Kulturmodells hinaus und führt einen neuen Begriff ein: New Public Commons (atarashii kōkyō). Das Konzept der New Public Commons kann als Grundlage für Solidarität dienen und es gewissenhaften Bürger/-innen ermöglichen, die Gesellschaft zu verbessern. Es kann auch als eine Sphäre fungieren, in der Menschen im Allgemeinen oder diejenigen, die an einer bestimmten Sache interessiert sind, freiwillig teilnehmen und ihre Sache fördern können. Japans Politik des lebenslangen Lernens seit den 2000 er Jahren wird in erster Linie zur Bildung und zum Wachstum dieser Öffentlichkeit beitragen.

Die laufende Initiative für lebenslanges Lernen wird voraussichtlich eine neue Art von staatlich gefördertem Disziplinarwissen hervorbringen, das die Schaffung der New Public Commons unterstützen kann. Das Wissen wird als "sōgōteki na chi" oder "umfassendes Wissen" bezeichnet, was der Zentralrat für Bildung als die Fähigkeit bezeichnet, Probleme zu identifizieren und flexibel zu bewerten. In einem globalen Kontext ist dieses Wissen identisch mit dem, was die OECD als "Schlüsselkompetenzen" definiert - wesentliche Fähigkeiten für die persönliche und soziale Entwicklung von Menschen in modernen, komplexen Gesellschaften. Dazu gehört die Fähigkeit, (1) in sozial heterogenen Gruppen zu interagieren, (2) autonom zu handeln und (3) Werkzeuge interaktiv einzusetzen. ${ }^{2}$ Insbesondere die zweite Fähigkeit ist am relevantesten, da sie Kompetenzen hervorruft, die es dem Einzelnen ermöglichen, sein Leben verantwortungsbewusst zu führen. Autonomes Handeln bedeutet "effektiv an der Entwicklung der Gesellschaft, an ihren sozialen, politischen und wirtschaftlichen Institutionen teilzunehmen und in verschiedenen Lebensbereichen gut zu funktionie- ren - am Arbeitsplatz, im persönlichen und familiären Leben sowie im bürgerlichen und politischen Leben « ${ }^{3}$. Um neue Kenntnisse und Fähigkeiten zur Verbesserung des bürgerlichen Lebens zu erwerben, wurden nach den neuesten Statistiken ${ }^{4} 24.238$ Kurse im Bereich Staatsbürgerschaft und Gemeinschaftssolidarität in der öffentlichen Bürgerhalle im ganzen Land angeboten.

\section{Sich am sozialen und politischen Leben beteiligen}

Mit diesen neuen Kenntnissen und Fähigkeiten, die durch die Kurse für lebenslanges Lernen erworben wurden, wird von den Menschen erwartet, dass sie spontan zu Aufgaben, wie der Festlegung von Tagesordnungen und der Problemlösung an der Basis, beitragen und so auf ein sich ständig änderndes soziales und politisches Leben reagieren können. Es wird auch erwartet, dass der japanische Staat entsprechende Themen für die Einrichtung der New Public Commons anbietet. Die Themen beziehen sich auf die Entwicklung einer selbstverantwortlichen Persönlichkeit. Interessanterweise wurde die Öffentlichkeit bei diesem Prozess im Rahmen der neuen Wachstumsstrategie, die 2010 von der Regierung von Premierminister Naoto Kan beschlossen wurde, beteiligt. Die Strategie bestand darin, eine starke Wirtschaft zu erreichen, indem der private, nichtstaatliche Sektor neue Anforderungen einbringen konnte.

Die New Public Commons wurden so insbesondere im Beschäftigungs- und Personalsektor gefördert, und 50\% der japanischen Bürger/-innen wurden schließlich durch zivilgesellschaftliche Organisationen wie die NPOs an der Entwicklung der New Public Commons beteiligt. $^{5}$

\section{Diskussionstechnik "jukugi"}

Darüber führte das Bildungsministerium die Kommunikationstechnik "jukugi" (Beratung) ein, die eine sorgfältige Diskussion und Betrachtung von Themen sowie die Suche nach 
Lösungen an der Basis beinhaltet. Die Praxis des jukugi lässt sich in erster Linie auf die Arbeit von Jürgen Habermas zur deliberativen Demokratie zurückführen ${ }^{6}$, die verschiedene Arten von politischen, administrativen, wirtschaftlichen und sozialen Institutionen sowohl im öffentlichen als auch im privaten Bereich umfasst. Basierend auf einer zweigleisigen Logik beginnen Diskussionen, wenn formelle Institutionen Themen aufwerfen, wobei informelle Beratungen außerhalb der Institutionen stattfinden und zu öffentlichen Meinungen werden, die dann die institutionelle Beratung beeinflussen. Formelle Verwaltungsinstitutionen spielen eine Rolle dabei, solche Fragen maßgeblich zu machen.

\section{Von "kakusa" zum New Public Commons}

Die Art des lebenslangen Lernens in Japan hat sich in letzter Zeit ständig verändert. Ich behaupte, dass die Förderung des lebenslangen Lernens den neoliberalen Staat beim Management der Regierung unterstützt. Es beinhaltet eine innovative Verteilung der Verantwortung zwischen Staat und Individuum durch Praktiken des lebenslangen Lernens. Mit anderen Worten, die Umsetzung der neuen Politik des lebenslangen Lernens seit den 2000er Jahren beinhaltete den Einsatz einer politischen Technik, die darauf abzielt, die derzeit geteilte und polarisierte japanische Bevölkerung allgemein bekannt als "kakusa" oder sozioökonomische Kluft - in das neue gewünschte Kollektiv als New Public Commons zu integrieren.

Der derzeitige politische Diskurs über den Neoliberalismus ist mehr als eine politische Verschiebung, die auf Deregulierung und Liberalisierung abzielt. Es bekräftigt die Interessen der Wirtschaftseliten und stellt einen direkteren Ausdruck der Klassenmacht wieder her. Vor dem Hintergrund der sich verschärfenden sozioökonomischen landesweiten Kluft in der Bevölkerung versucht der neoliberale japanische Staat bei der Verwirklichung der New Public Commons, das Risiko einer Regierungsführung mit der Einführung einer starken Initiative für lebenslanges Lernen zu bewältigen.

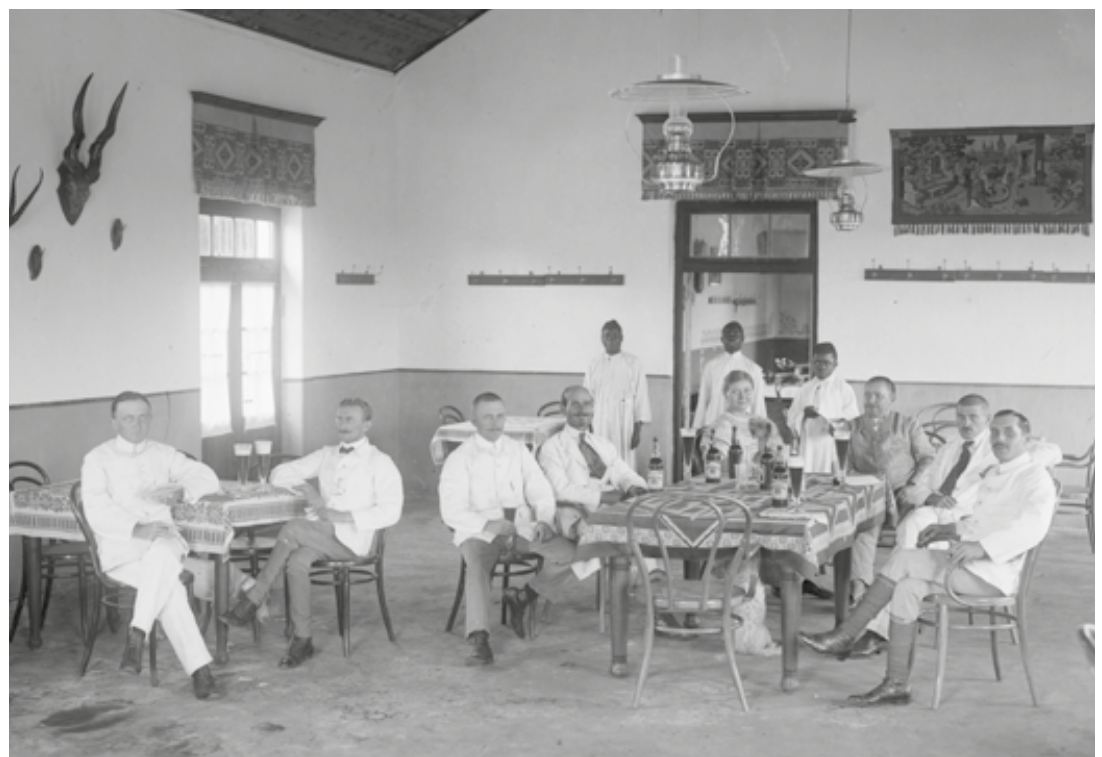

Was treiben wir Deutschen in Afrika? Deutsch-Ostafrika: Siedler/-innen in einer Gastwirtschaft mit Jagdtrophäen und Bediensteten

Quelle: Bundesarchiv / Nachlass Walther Dobbertin

"Umfassendes Wissen", das durch staatlich geförderte Aktivitäten zum lebenslangen Lernen generiert wird, ist disziplinarischer Natur. Die Entwicklung dieses Wissens durch lebenslanges Lernen kann Teil der Bemühungen des neoliberalen Staates zum Kapazitätsaufbau sein, indem nicht-staatliche Sektoren bei der Bereitstellung von Bildung wiederbelebt werden. ${ }^{7}$ Dieses Wissen soll den Bürger/-innen helfen, Probleme ausschließlich in lokalen Gemeinschaften zu lösen - den täglichen Zentren des Lebens, der Gedanken und des Verhaltens der Menschen. Tatsächlich wurden lokale Gemeinschaften als Orte für die Ausübung von Aktivitäten des lebenslangen Lernens institutionalisiert, sodass soziale Solidarität und kollektives Bewusstsein unter den Menschen wachsen würden, indem sie sich als aktive Mitglieder der Gemeinschaft an der Problemlösung beteiligen.

Eine wichtige neoliberale Prämisse ist hier, dass jukugi-Teilnehmer/-innen selbstbestimmte Individuen sind. Die Themen werden zu einem integralen Bestandteil der Festigung des Neoliberalismus, der die Wohlfahrtsstaatlichkeit ersetzt und damit die Tugenden der Selbstverantwortung feiert. Sie helfen auch Einzelpersonen, ein unabhängiges, unternehmerisches Selbst zu werden, das das notwendige Wissen aufbauen kann, um positiv an der Gesellschaft teilzunehmen und die neoliberale Politik strategisch zu gestalten.

\section{Junge und Alte lernen in Gemeinschaftsschulen}

Um meine Argumentation über die Rolle des lebenslangen Lernens in der neoliberalen Regierungspolitik lebenslangen Lernens zu erweitern, seien Japans jüngste Bemühungen erwähnt, die "Gemeinschaftsschulen" in das öffentliche Bildungssystem zu integrieren. Ab Anfang der 2000er Jahre wurden landesweit immer mehr Gemeindeschulen an der Basis entwickelt. Diese Schulen fungieren als Zentren für gemeinschaftliche Lösungen, was bedeutet, dass die betroffenen Parteien gemeinsam die Initiative ergreifen, um selber die anstehenden Probleme zu lösen, statt sie in einer passiven Mentalität der Verwaltung oder dem Markt zu überlassen. Darüber hinaus ist die Einrichtung öffentlicher Schulen als lokale Gemeinde- 
zentren ein Schritt in Richtung der Integration von formaler Schulbildung und non-formaler, gemeinschaftsorientierter sozialer Bildung. Das formelle Schulsystem wird auf der Grundlage der reichen japanischen Tradition der sozialen Bildung wieder aufgebaut. Derzeit gibt es in Japan 5.432 Gemeinschaftsschulen, eine Steigerung von 3.600 im Vergleich zum Vorjahr. ${ }^{8}$ Dies liegt hauptsächlich daran, dass das Bildungsministerium von der Schulbehörde auf kommunaler Ebene die Einrichtung von Gemeinschaftsschulen als obligatorische Anstrengung verlangt.

\section{Schulen: Lernorte auch für Erwachsene}

Lokale Schulen, die tief in der lokalen Gemeinschaft verwurzelt sind, sind heutzutage zu einem Zentrum für das lebenslange Lernen geworden. Kinder lernen durch ihre Kommunikation mit Erwachsenen, wie sie in dieser schwierigen und komplizierten neoliberalen Welt bestehen können. Für Erwachsene führt eine solche Mitwirkung an örtlichen Schulen zu eigenem Lernen. Die örtlichen Schulen würden zu einem
Lernort für Erwachsene, nicht nur für Lehrer/-innen, sondern auch für Eltern und Anwohner/-innen. Lehren wird zum Lernen. Von Erwachsenen wird erwartet, dass sie als Assistenten/-innen für reguläre Lehrkräfte in Klassenzimmern und in außerschulischen Clubs am Unterricht teilnehmen. Sie könnten auch Gastlehrer/-innen werden, wenn sie sich auf bestimmte Bereiche spezialisiert haben. Sie lernen wiederrum selber durch inre Unterrichtsvorbereitungen und Reflexionen nach dem Unterricht. Die örtliche Schule wird voraussichtlich auch eine wichtige Rolle in der Erwachsenenbildung spielen. Letztendlich würde eine solche lokale Zusammenarbeit die Schule als Zentrum für Gemeinschaftslösungen wiederbeleben und zur Schaffung der New Public Commons beitragen. Von verschiedenen Interessengruppen in der Gemeinde wird erwartet, dass sie die örtlichen Schulen nutzen und dazu beitragen, die Gemeinde aufzubauen. Das lebenslange Lernen ist in jüngster Zeit in den lokalen Gemeinschaften immer tiefer verankert, da Japan auf dem Weg zu einem neuen Lernstil ist.

\section{Anmerkungen}

1 Ogawa 2016.

2 Rychen 2003, S. 85-104.

3 Ebd. S. 91.

4 MEXT (Ministerium für Bildung, Kultur, Sport, Wissenschaft und Technologie) 2017.

5 Cabinet Office 2009.

6 Habermas 1996.

7 Siehe Mok 2007

8 MEXT 2018.

\section{Literatur}

Habermas, J. (1996): Theorie des kommunikativen Handels. Frankfurt am Main.

Mok, K. H. (2007): Questing for internationalization of universities in Asia: Critical reflections. In: Journal of Studies in International Education 11 (3/4), S. 433-454.

Ogawa, A. (2016): ,Lifelong Learning in Neoliberal Japan: Risk, Community, and Knowledge. New York 2016

Rychen, D. S. (2003): Key competencies: Meeting important challenges in life. In: Rychen, D. S.; Salganik, L. H. (Hg.): Key competencies for a successful life and well-functioning society. Cambridge, Toronto, Bern, Göttingen, S. $63-107$

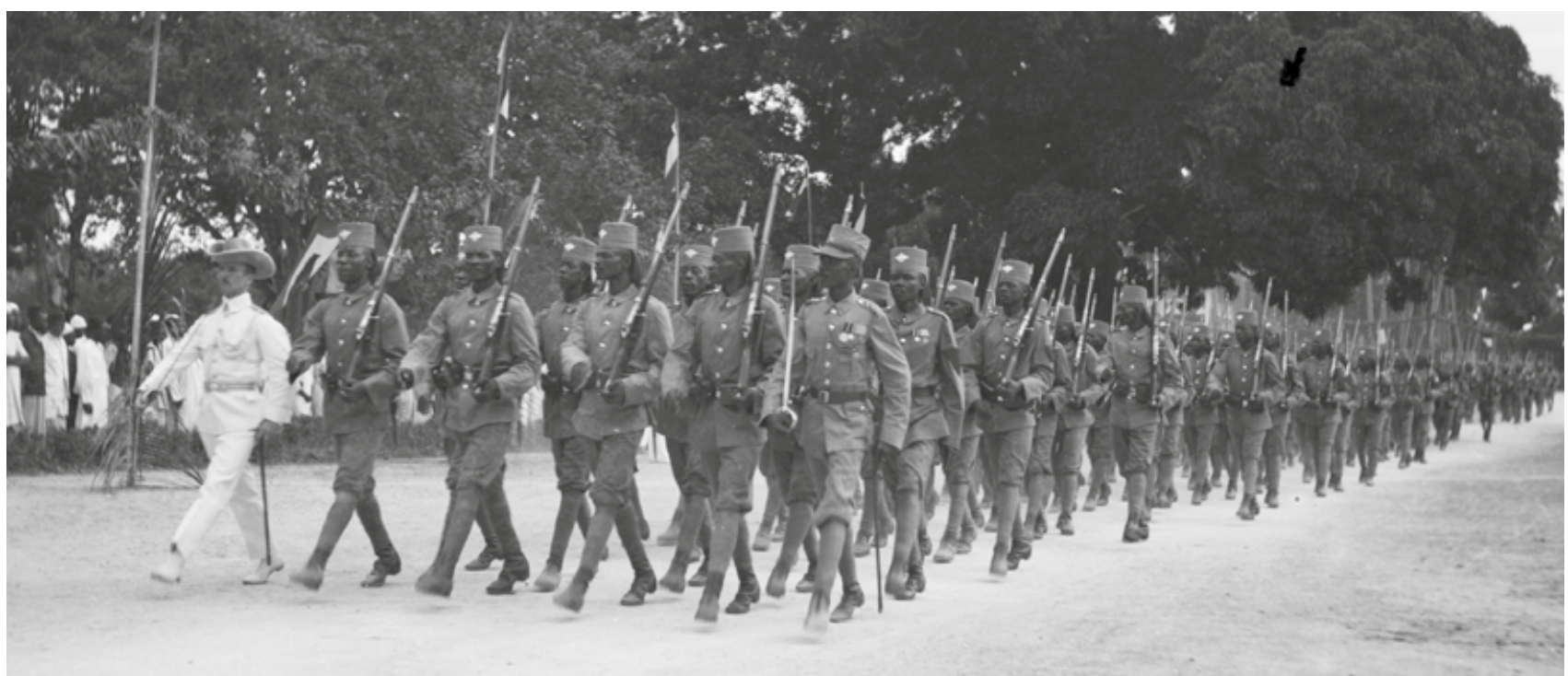

Was treiben wir Deutschen in Afrika? Deutsch-Ostafrika: Daressalam - Schutztruppe und angetretene Askaris bei der 


\section{University Library}

\section{- M M N E R VA A gateway to Melbourne's research publications}

Minerva Access is the Institutional Repository of The University of Melbourne

Author/s:

Ogawa, A

Title:

Sh\#gai gakush\# - lebenslanges Lernen in Japan

Date:

2020-05-04

Citation:

Ogawa, A. (2020). Sh\#gai gakush\# - lebenslanges Lernen in Japan. Erwachsenenbildung, 66 (2), pp.59-62. https://doi.org/10.13109/erbi.2020.66.2.59.

Persistent Link:

http://hdl.handle.net/11343/258619

License:

CC BY-NC-SA 